\title{
Attractors in Frictional Systems Subjected to Periodic Loads
}

\author{
Lars-Erik Andersson, J R. Barber and Y-J Ahn
}

\section{Linköping University Post Print}

\section{Tweet}

N.B.: When citing this work, cite the original article.

Original Publication:

Lars-Erik Andersson, J R. Barber and Y-J Ahn, Attractors in Frictional Systems Subjected to Periodic Loads, 2013, SIAM Journal on Applied Mathematics, (73), 3, 1097-1116.

http://dx.doi.org/10.1137/120885024

Copyright: Society for Industrial and Applied Mathematics

http://www.siam.org/

Postprint available at: Linköping University Electronic Press

http://urn.kb.se/resolve?urn=urn:nbn:se:liu:diva-95982 


\title{
ATTRACTORS IN FRICTIONAL SYSTEMS SUBJECTED TO PERIODIC LOADS*
}

\author{
L.-E. ANDERSSON ${ }^{\dagger}$, J. R. BARBER ${ }^{\ddagger}$, AND Y.-J. AHN ${ }^{\S}$
}

\begin{abstract}
This paper explores the effect of initial conditions on the behavior of coupled frictional elastic systems subject to periodic loading. Previously, it has been conjectured that the long term response will be independent of initial conditions if all nodes slip at least once during each loading cycle. Here, this conjecture is disproved in the context of a simple two-node system. Counter examples are presented of "unstable" steady-state orbits that repel orbits starting from initial conditions that are sufficiently close to the steady state. The conditions guaranteeing stability of such steady states are shown to be more restrictive than those required for the rate problem to be uniquely solvable for arbitrary derivative of the external loading. In cases of instability, the transient orbit is eventually limited either by slip occurring at both nodes simultaneously, or by one node separating. In both cases a stable limit cycle is obtained. Depending on the slopes of the constraint lines, the limit cycle can involve two periods of the loading cycle, in which case it appears to be unique, or it may repeat every loading cycle, in which case distinct limit cycles are reached depending on the sign of the initial deviation from the steady state. In the case of instability an example is given of a loading for which a quasi-static evolution problem with multiple solutions exists, whereas all rate problems are uniquely solvable.
\end{abstract}

Key words. contact problems, shakedown, Melan's theorem, Coulomb friction, attractors, uniqueness

AMS subject classifications. 34D05, 34D20, 70J25

DOI. $10.1137 / 120885024$

1. Introduction. Many engineering systems comprise a set of elastic components assembled using frictional interfaces such as bolted joints. These interfaces are usually intended to prevent relative motion, but since the contacting bodies are deformable, it is often difficult to prevent the occurrence of microslip -i.e., a frictional slip that occurs in some localized region(s), while the rest of the interface remains stuck (Johnson [7]). Most systems of this kind will be subject to some level of vibration, causing the interfaces to experience periodic loading superposed on the constant load associated with the joint clamping force. In this case, microslip leads to the dissipation of energy in the form of heat (Wentzel [11]) and can cause the development and propagation of fretting fatigue cracks (Nowell, Dini, and Hills [10]).

Previous studies of elementary discrete elastic systems with frictional interfaces have shown that the steady state (and in particular the steady-state energy dissipation) can depend on the initial condition when there is elastic coupling between the normal and tangential contact problems (Klarbring, Ciavarella, and Barber [9] and Ahn, Bertocchi, and Barber [2]). However, in a related study of the frictional dissipation in an elastic body containing multiple microcracks, Jang and Barber [6] found that this history dependence occurred only for relatively moderate values of the

\footnotetext{
*Received by the editors July 18, 2012; accepted for publication (in revised form) February 14, 2013; published electronically May 9, 2013.

http://www.siam.org/journals/siap/73-3/88502.html

${ }^{\dagger}$ Department of Mathematics, Linköping University, SE-581 83 Linköping, Sweden (lars-erik. andersson@liu.se).

${ }^{\ddagger}$ Department of Mechanical Engineering, University of Michigan, Ann Arbor, MI 48109-2125 (jbarber@engin.umich.edu).

§Samsung SDI Central Research Center, Gongse-dong, Giheung-gu, Yongin-si, Gyeonggi-do, Korea (yjahn70@gmail.com).
} 
periodic load amplitude. For larger amplitudes, the system appeared to converge on a unique steady state.

A possible explanation for this behavior was advanced by Barber [5], who conjectured that the steady state would be unique if the amplitude of the periodic load were sufficient to ensure that all nodes in the discrete system slip at least once during each period. A heuristic argument for this conjecture is that dependence on initial conditions depends on the system possessing "memory," and this memory essentially resides at any given time in the tangential displacements of the nodes that are not slipping, since these nodes could be located anywhere in a finite space bounded by the appropriate friction cone. Now if all nodes slip at least once during each cycle (but not necessarily all at the same time), the memory of the initial conditions must be passed from node to node during the cycle, and there is every reason to expect that this will lead to a degradation of memory over time. In the present paper, we shall examine the implications and limitations of this conjecture in the context of the simple two-node two-dimensional system used by Ahn, Bertocchi, and Barber [2].

2. Ahn's slip-displacement diagram. Ahn, Bertocchi, and Barber [2] showed that the evolution of a discrete two-dimensional frictional elastic system could most conveniently be characterized by tracking the motion of the system in $\boldsymbol{v}$-space, where $v_{i}$ is the tangential (slip) displacement of the $i$ th contact node. Since the support structure is linear elastic, the vectors of normal and tangential nodal contact forces, $\boldsymbol{p}, \boldsymbol{q}$, respectively, must be related to the vectors of normal and tangential nodal displacements $\boldsymbol{w}, \boldsymbol{v}$ by equations of the form

$$
\left\{\begin{array}{l}
\boldsymbol{q} \\
\boldsymbol{p}
\end{array}\right\}=\left\{\begin{array}{l}
\boldsymbol{q}^{w} \\
\boldsymbol{p}^{w}
\end{array}\right\}+\left[\begin{array}{cc}
\boldsymbol{A} & \boldsymbol{B}^{T} \\
\boldsymbol{B} & \boldsymbol{C}
\end{array}\right]\left\{\begin{array}{c}
\boldsymbol{v} \\
\boldsymbol{w}
\end{array}\right\}
$$

where $\boldsymbol{q}^{w}, \boldsymbol{p}^{w}$ are the contact forces that would be produced if the nodes were all welded in contact with $v_{i}=w_{i}=0$. Notice that

$$
\boldsymbol{K}=\left[\begin{array}{cc}
\boldsymbol{A} & \boldsymbol{B}^{T} \\
\boldsymbol{B} & \boldsymbol{C}
\end{array}\right]
$$

is the reduced stiffness matrix for the system and hence must be symmetric and positive definite. It follows that $\boldsymbol{A}, \boldsymbol{C}$ must also be symmetric and positive definite, but no such restriction applies to the matrix $\boldsymbol{B}$ which defines the coupling between normal and tangential effects.

Equations (2.1) define the linear relations that must hold between the contact forces $q_{i}, p_{i}$ and the corresponding nodal displacements $v_{j}, w_{j}$ by virtue of the linearelastic nature of the supporting structure. However, these forces must also satisfy the inequalities associated with the Coulomb friction law. In particular, if all the nodes are in contact $(\boldsymbol{w}=0)$, the frictional inequality governing (say) negative $\operatorname{slip}\left(\dot{v}_{i}<0\right)$ at node $i$ is $q_{i} \leq \mu_{i} p_{i}$, where $\mu_{i}$ is the corresponding coefficient of friction and the dot denotes the derivative with respect to time $t$. Here, we adopt the convention that compressive normal tractions are positive. Using (2.1), we can express this condition in terms of $\boldsymbol{v}$ as

$$
\sum_{j=1}^{N}\left(a_{i j}-\mu_{i} b_{i j}\right) v_{j} \leq \mu_{i} p_{i}^{w}-q_{i}^{w},
$$

which restricts $\boldsymbol{v}$ to one side of a certain hyperplane in $\boldsymbol{v}$-space. As the external loads vary periodically in time, the right-hand side of this inequality changes, indicating 


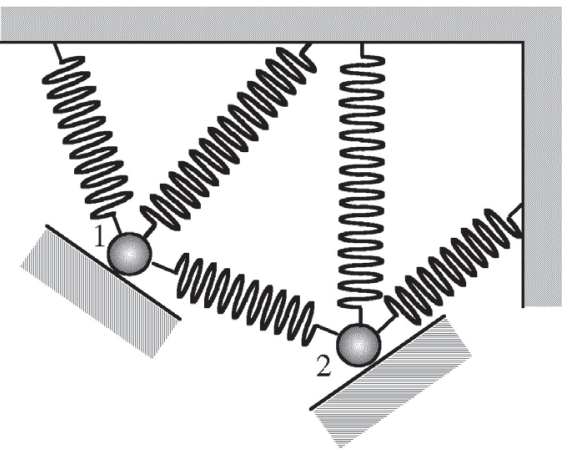

(a)

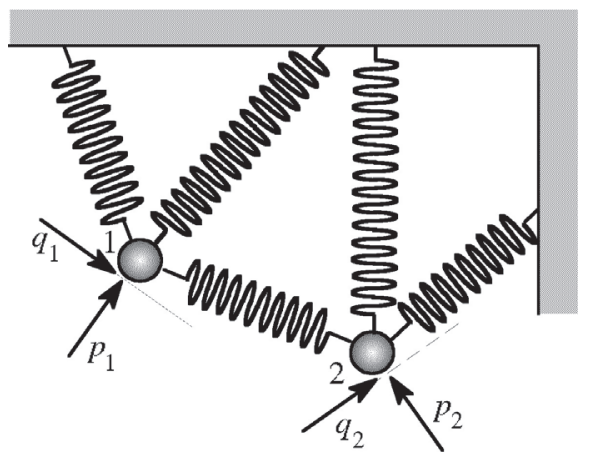

(b)

FIG. 1. (a) A two-node frictional elastic system, and (b) sign conventions for the nodal contact forces $q_{i}, p_{i}$.

that the corresponding hyperplane moves in the space while retaining the same normal (defined by the stiffness matrix and the friction coefficients). Each node contributes two such hyperplanes (one for positive and one for negative slip), and their motion during the loading cycle "pushes" the point $\boldsymbol{v}$ in accordance with the frictional flow rule at the corresponding node.

This procedure is best explained in the context of a simple system with just two contact nodes, which can be visualized as two particles connected to ground and to each other by generalized linear springs as illustrated in Figure 1(a), though equally it could represent a finite element discretization of a continuum problem in which the contact region contains only two nodes. Sign conventions for the nodal contact forces $q_{i}, p_{i}$ are shown in Figure 1(b), and similar directions are taken for the nodal displacements $v_{i}, w_{i}$, so that (for example) a positive value of $w_{i}$ indicates that a positive gap opens up between the node and the obstacle. For this system, the inequalities (2.3) define just the four frictional constraints

$$
\begin{aligned}
& \left(a_{11}-\mu_{1} b_{11}\right) v_{1}+\left(a_{12}-\mu_{1} b_{12}\right) v_{2} \leq \mu_{1} p_{1}^{w}-q_{1}^{w}, \quad \mathcal{O}_{-}^{1}, \\
& \left(a_{11}+\mu_{1} b_{11}\right) v_{1}+\left(a_{12}+\mu_{1} b_{12}\right) v_{2} \geq-\mu_{1} p_{1}^{w}-q_{1}^{w}, \quad \mathcal{O}_{+}^{1}, \\
& \left(a_{21}-\mu_{2} b_{21}\right) v_{1}+\left(a_{22}-\mu_{2} b_{22}\right) v_{2} \leq \mu_{2} p_{2}^{w}-q_{2}^{w}, \quad \mathcal{O}_{-}^{2}, \\
& \left(a_{21}+\mu_{2} b_{21}\right) v_{1}+\left(a_{22}+\mu_{2} b_{22}\right) v_{2} \geq-\mu_{2} p_{2}^{w}-q_{2}^{w}, \quad \mathcal{O}_{+}^{2},
\end{aligned}
$$

which control the motions $\mathcal{O}_{-}^{1}: \dot{v}_{1}<0 ; \mathcal{O}_{+}^{1}: \dot{v}_{1}>0 ; \mathcal{O}_{-}^{2}: \dot{v}_{2}<0 ; \mathcal{O}_{+}^{2}: \dot{v}_{2}>0$. For more detail on the way in which these constraints govern the motion of the point $P\left(v_{1}, v_{2}\right)$ under periodic loading, the reader is referred to Ahn, Bertocchi, and Barber $[2]$.

3. A restricted class of loading scenarios. Initially we restrict our attention to the class $\mathcal{C}_{1}$ defined as follows.

Definition 3.1. The class of loading scenarios $\mathcal{C}_{1}$ is such that there exists a steady-state periodic orbit in which

(i) each of the constraints in (2.4) is active once and only once per cycle, and

(ii) there exists no point in the orbit at which more than one constraint is active.

In physical terms, condition (ii) means that the two nodes never slip at the same time, and neither goes into a state of separation, since incipient separation corresponds to the case where the two constraints for the separating node are both active.

Copyright (c) by SIAM. Unauthorized reproduction of this article is prohibited. 


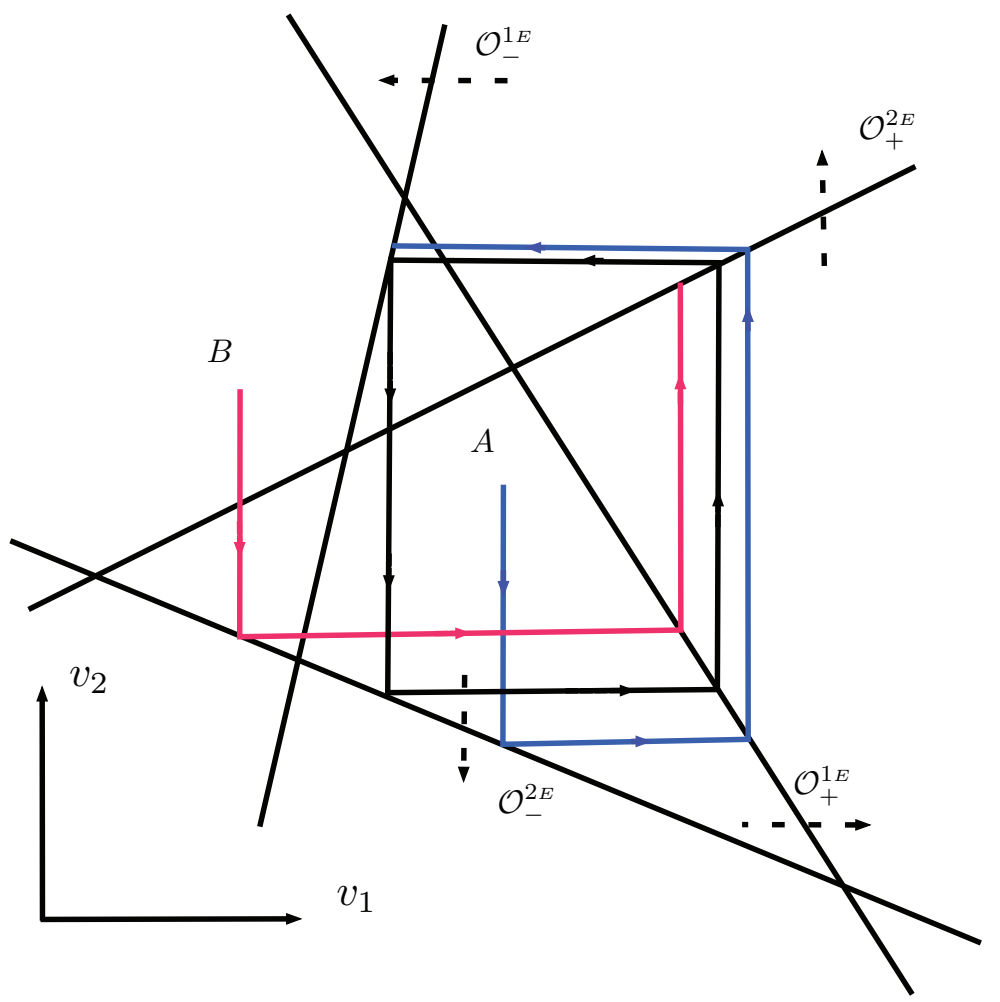

FIG. 2. Convergence of a two-node system on a unique steady state. The steady-state periodic orbit (black) attracts the red and blue orbits (see electronic version of article for color images).

This situation can be realized by maintaining the external normal tractions $p_{1}^{w}, p_{2}^{w}$ constant and varying the tangential tractions in the form

$$
q_{1}^{w}=\bar{q}_{1}+\hat{q}_{1} \cos (\omega t), \quad q_{2}^{w}=\bar{q}_{2}+\hat{q}_{2} \sin (\omega t) .
$$

In other words, the tangential normal tractions oscillate periodically out of phase with each other. If the amplitudes $\hat{q}_{i}$ are suitably chosen, it is easily contrived that slip at node $i$ occurs only when $q_{i}^{w}(t)$ is near its maximum or minimum (i.e., near $\omega t=0, \pi$ for node 1 and $\omega t=\pi / 2,3 \pi / 2$ for node 2 ) and hence that there are no occasions when both nodes slip simultaneously.

A simpler scenario in class $\mathcal{C}_{1}$ that is useful for illustrative purposes is to select time-varying functions $p_{i}^{w}, q_{i}^{w}$ such that the right-hand sides of conditions (2.4) remain constant and located at a "rest" position that allows a range of values $\boldsymbol{v}$, except that in each loading cycle, the constraints advance and recede one at a time.

A typical scenario of this latter form is shown in Figure 2, where the four constraints advance and recede in the sequence $\mathcal{O}_{-}^{2}, \mathcal{O}_{+}^{1}, \mathcal{O}_{+}^{2}, \mathcal{O}_{-}^{1}, \ldots$ In this figure, the lines $\mathcal{O}_{+}^{1 E}, \mathcal{O}_{-}^{1 E}, \mathcal{O}_{+}^{2 E}, \mathcal{O}_{-}^{2 E}$ represent the extreme positions of the four constraints (i.e., the positions that exclude the maximum region of the space).

Starting from the initial condition $A$, the external loading causes $\mathcal{O}_{-}^{2}$ to move downwards. When this line reaches the point $A$, negative slip $\left(\dot{v}_{2}<0\right)$ commences at node 2 and continues until $\mathcal{O}_{-}^{2}$ reaches its extreme position $\mathcal{O}_{-}^{2 E}$. The constraint then recedes and both nodes remain stuck until the loading causes $\mathcal{O}_{+}^{1}$ to advance. This 
time, positive slip $\left(\dot{v}_{1}>0\right)$ commences at node 1 when $\mathcal{O}_{+}^{1}$ reaches the instantaneous point $\left(v_{1}, v_{2}\right)$ and continues until this constraint reaches its extreme value $\mathcal{O}_{+}^{1 E}$. With this sequence of loading, it is clear that the transient trajectory will comprise an alternating set of horizontal and vertical motions in Figure 2, with each segment terminating at a point on the extreme position of the relevant active constraint.

In Figure 2, we illustrate this transient evolution process from two initial conditions, $A$ and $B$, and demonstrate that each converges asymptotically on a unique periodic rectangular orbit defined by the black line.

The transient trajectory and the periodic rectangular orbit depend upon the slopes of the constraint lines, which we define through the angles $\alpha_{i}, \beta_{i}$ in Figure 3.

The sign convention is chosen such that $\alpha_{j}$ is measured clockwise from the direction of slip governed by the constraint to the constraint line, while $\beta_{j}$ is measured counterclockwise from the same direction of slip to the other constraint line at the given corner. Notice that with this convention, all the angles $\beta_{j}$ are positive in Figure 3 , except at the top left corner, where $\beta_{2}<0$.

We also note from this figure that

$$
\alpha_{i}=\frac{\pi}{2}-\beta_{i-1}, \quad \text { implying } \quad \cos \alpha_{i}=\sin \beta_{i-1} \quad(\bmod 4) .
$$

The angles $\alpha_{i}, \beta_{i}$ cannot be chosen arbitrarily, since they must be defined in terms of the stiffness matrices $\boldsymbol{A}, \boldsymbol{B}$ of section 2. If we denote the $i$ th row vectors of the matrices $\boldsymbol{A}$ and $\boldsymbol{B}$ by $\boldsymbol{a}^{i}$ and $\boldsymbol{b}^{i}$, respectively, then the normals of the obstacles $\mathcal{O}_{ \pm}^{i}$ are

$$
\boldsymbol{n}_{ \pm}^{i}=\boldsymbol{a}^{i} \pm \mu_{i} \boldsymbol{b}^{i}=\left(a_{i 1} \pm \mu_{i} b_{i 1}, a_{i 2} \pm \mu_{i} b_{i 2}\right), \quad i=1,2
$$

see also (2.4) and Figure 3.

By comparison with the constraint conditions (2.4), it can be shown that the angles $\beta_{i}$ take the values

$$
\begin{aligned}
& \beta_{1}=\arctan \left(\frac{a_{12}+\mu_{1} b_{12}}{a_{11}+\mu_{1} b_{11}}\right), \quad \beta_{2}=-\arctan \left(\frac{a_{21}-\mu_{2} b_{21}}{a_{22}-\mu_{2} b_{22}}\right), \\
& \beta_{3}=\arctan \left(\frac{a_{12}-\mu_{1} b_{12}}{a_{11}-\mu_{1} b_{11}}\right), \quad \beta_{4}=-\arctan \left(\frac{a_{21}+\mu_{2} b_{21}}{a_{22}+\mu_{2} b_{22}}\right) .
\end{aligned}
$$

The matrix $\boldsymbol{A}$ must be symmetric and positive definite, but we must also impose the following restriction on the coefficients of friction $\mu_{i}$ to ensure that the rate problem is well-posed [8].

CONDITION 3.1. The necessary and sufficient condition for the rate problem to have a unique solution for all time-derivatives of loads is that all matrices with elements

$$
a_{i j} \pm \mu_{i} b_{i j}
$$

i.e., all matrices

$$
A+\mathcal{M E B}
$$

with $\mathcal{E}=\operatorname{diag}\left\{\varepsilon_{1}, \varepsilon_{2}\right\}$ and $\mathcal{M}=\operatorname{diag}\left\{\mu_{1}, \mu_{2}\right\}$ and $\varepsilon_{i}= \pm 1, i=1,2$, should be $P$ matrices, i.e., that they should have all principal subdeterminants positive. In other 


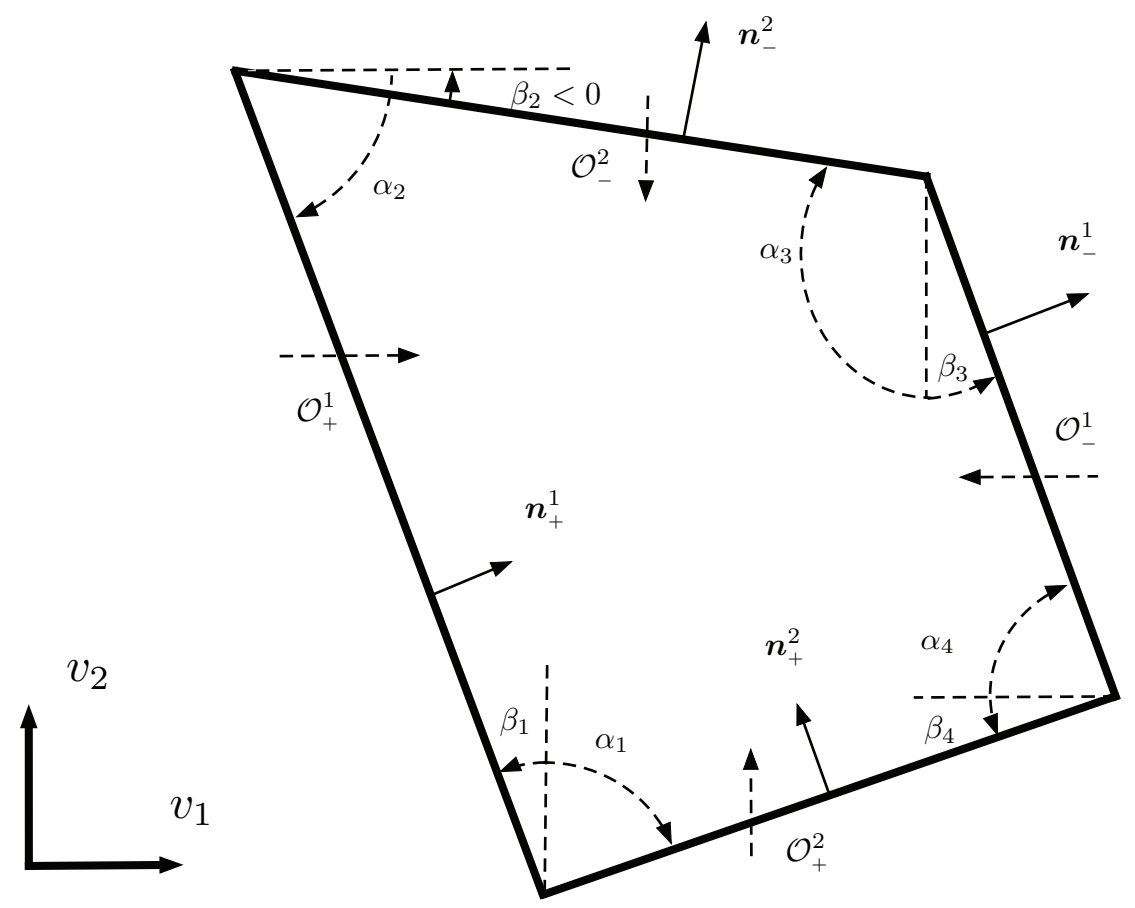

FIG. 3. Definition of the angles $\alpha_{i}, \beta_{i}$.

words

$$
\begin{aligned}
& a_{11}+\varepsilon_{1} \mu_{1} b_{11}>0, \quad a_{22}+\varepsilon_{2} \mu_{2} b_{22}>0, \\
& \left(a_{11}+\varepsilon_{1} \mu_{1} b_{11}\right)\left(a_{22}+\varepsilon_{2} \mu_{2} b_{22}\right)-\left(a_{12}+\varepsilon_{1} \mu_{1} b_{12}\right)\left(a_{21}+\varepsilon_{2} \mu_{2} b_{21}\right)>0 .
\end{aligned}
$$

It is straightforward to demonstrate that these conditions are equivalent to

$$
\begin{aligned}
& -\frac{\pi}{2}<\beta_{i}<\frac{\pi}{2}, \quad 0<\alpha_{i}<\pi, \\
& 0<\alpha_{i}+\beta_{i}<\pi,
\end{aligned}
$$

where $i=1,2,3,4$. These inequalities define the shaded hexagonal region $A B C D E F$ in Figure 4.

4. Convergence criterion. Suppose there exists a steady-state orbit $\boldsymbol{v}^{\mathrm{ss}}(t)$ and that during some transient trajectory the slip displacements are defined by $\boldsymbol{v}(t)$. Recalling that only one node is allowed to slip at a given time $t$, we define a measure $\Delta$ of the deviation from the steady state such that

$$
\begin{aligned}
& \Delta=v_{1}(t)-v_{1}^{s s}(t) \quad \text { if } \quad \dot{v}_{2} \neq 0 \\
& =v_{2}(t)-v_{2}^{s s}(t) \quad \text { if } \quad \dot{v}_{1} \neq 0 \text {. }
\end{aligned}
$$

Figure 5 represents a phase $t<t_{1}$ of positive slip at node 2 under the influence of constraint $\mathcal{O}_{+}^{2}$. If the next phase $t>t_{1}$ involves slip at node 1 , we see that with the definition (4.1)

$$
\Delta^{+}=\Delta^{-} \tan \beta_{4}
$$

Copyright (C) by SIAM. Unauthorized reproduction of this article is prohibited. 


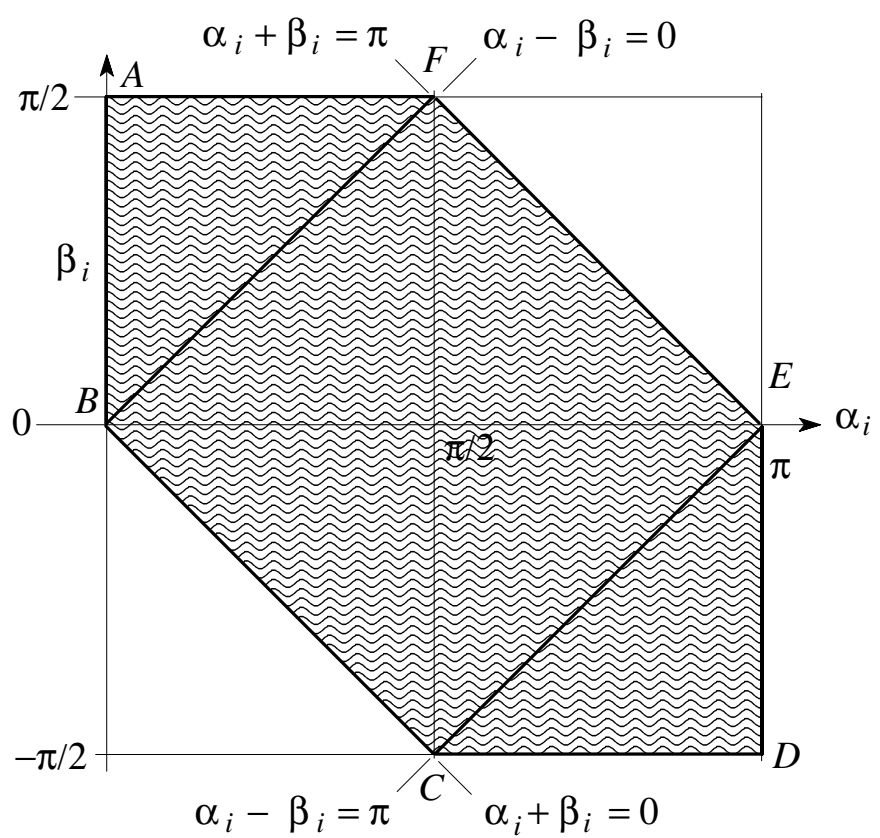

FIG. 4. Regions of $\alpha-\beta$-space satisfied by (3.6) and (3.7) (shaded). The condition (4.6) $)^{2}$ below is satisfied only if all four points lie in the central square BCEF.

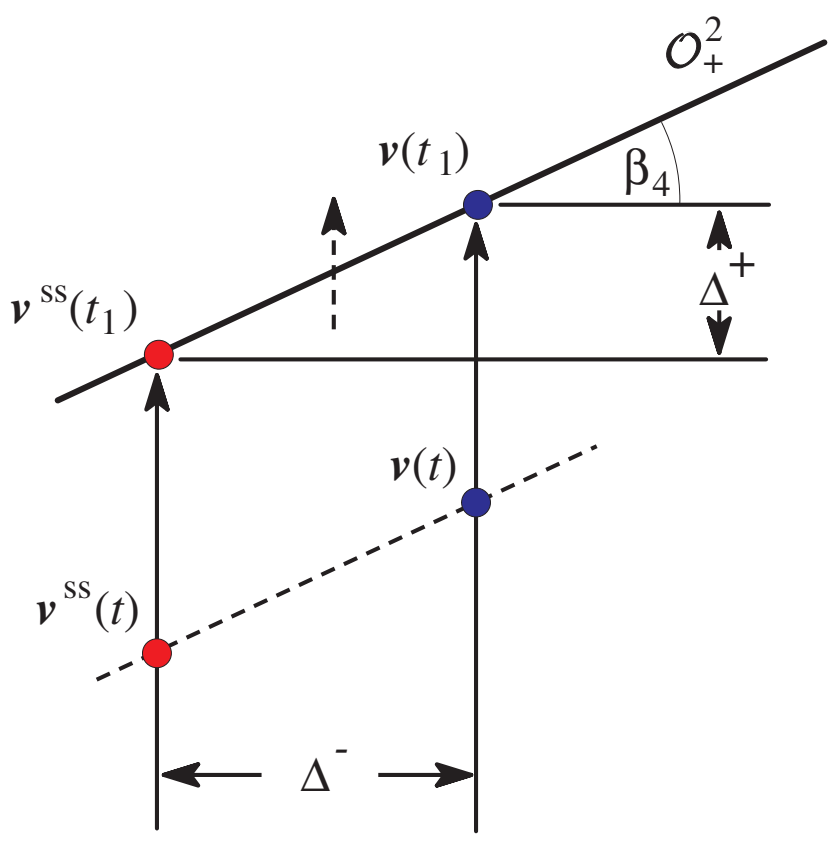

FIG. 5. Transition from slip at node 2 to slip at node 1. 
Similar arguments can be applied to the remaining constraints, leading to the results

$$
\begin{array}{llrl}
\Delta^{+} & =-\Delta^{-} \tan \beta_{1}, & & \mathcal{O}_{+}^{1}, \\
\Delta^{+} & =-\Delta^{-} \tan \beta_{3}, & & \mathcal{O}_{-}^{1}, \\
\Delta^{+} & =\Delta^{-} \tan \beta_{4}, & & \mathcal{O}_{+}^{2}, \\
\Delta^{+} & =\Delta^{-} \tan \beta_{2}, & & \mathcal{O}_{-}^{2},
\end{array}
$$

where the constraint referred to is that active before the transition.

We conclude that after a full cycle in Figure 2, the distance $\Delta$ has been multiplied by a factor

$$
\mathcal{R}=\prod_{1 \leq i \leq 4}\left(\tan \beta_{i}\right)
$$

Also, after $m$ cycles this distance is multiplied by a factor

$$
\prod_{1 \leq i \leq 4}\left(\tan \beta_{i}\right)^{m}
$$

An alternative form of (4.3) can be obtained by using (3.1), giving

$$
\mathcal{R}=\prod_{1 \leq i \leq 4}\left(\frac{\cos \alpha_{i}}{\cos \beta_{i}}\right)
$$

We conclude that if the loading is such that a periodic orbit $\boldsymbol{v}^{\mathrm{ss}}(t)$ belonging to class $\mathcal{C}_{1}$ exists, then this orbit will attract all other orbits $\boldsymbol{v}(t)$ having an initial position $\boldsymbol{v}(0)$ sufficiently close to $\boldsymbol{v}^{\mathrm{ss}}(0)$, i.e., $\left(\boldsymbol{v}(t)-\boldsymbol{v}^{\mathrm{ss}}(t)\right) \rightarrow \mathbf{0}$ as $t \rightarrow \infty$, if and only if $|\mathcal{R}|<1$. Moreover in such cases, the convergence is exponential in time.

We also note that a sufficient condition for $|\mathcal{R}|<1$ is

$$
\mathcal{R}_{i}^{(1)}=\left|\tan \beta_{i}\right|<1 \quad \text { for all } i \quad \text { or } \quad \mathcal{R}_{i}^{(2)}=\left|\frac{\cos \alpha_{i}}{\cos \beta_{i}}\right|<1 \quad \text { for all } i
$$

5. Restrictions on the values of $\boldsymbol{\beta}_{\boldsymbol{i}}$. We first note that the ratio $\mathcal{R}_{i}^{(2)}$ defined in (4.6) is less than unity for points located in the square BCEF in Figure 4, but in the two triangles $A B F$ and $C D E$, we have $\mathcal{R}_{i}^{(2)}>1$. In other words, there are some values of the angles $\alpha_{i}, \beta_{i}$ that satisfy Condition 3.1, but which lead to an increase in the deviation of the transient trajectory from the periodic orbit, suggesting the possibility of orbits of class $\mathcal{C}_{1}$ that repel other orbits.

To explore this question further, we note that the definitions (3.2), (3.3) imply that the angles $\beta_{1}, \beta_{3}$ will have the same sign if and only if $\left|\mu_{1} b_{12}\right|<\left|a_{12}\right|$, and that the sign in this case will be that of $a_{12}$. Similarly, $\beta_{2}, \beta_{4}$ will have the same sign if and only if $\left|\mu_{2} b_{21}\right|<\left|a_{12}\right|$, and the sign in this case will be that of $-a_{12}$. Thus, it is impossible for all four values $\beta_{i}$ to have the same sign. The only remaining possibilities are (1) that two angles are positive and two negative, or (2) that three are positive and one negative, or vice versa. These two cases exhibit significant qualitative differences and will be considered separately. 
5.1. Two positive and two negative angles. We first note that the inequalities (3.4), (3.5) imply that

$$
-\tan \beta_{i} \tan \beta_{i+1}<1 \quad(\bmod 4) .
$$

If (for example) $\beta_{1}>0, \beta_{2}>0, \beta_{3}<0, \beta_{4}<0$, two of these inequalities will be satisfied identically (i.e., for all values of $\beta_{i}$ of the stated sign), but the remaining two can then be combined to establish the condition

$$
0<\prod_{1 \leq i \leq 4}\left(\tan \beta_{i}\right)<1 .
$$

If instead we have $\beta_{1}>0, \beta_{2}<0, \beta_{3}>0, \beta_{4}<0$ (the only qualitatively different case), none of the inequalities are satisfied identically, but a similar combination leads to $(5.2)$ as before. In view of (4.3), we conclude that periodic orbits of class $\mathcal{C}_{1}$ will always attract other orbits, provided any two of the four angles $\beta_{i}$ are positive and the other two negative.

We also note that for this case $\mathcal{R}>0$, implying that phases of positive slip at node 1 (for example) in successive cycles both lie on the same side of the steady state orbit. In other words, the asymptotic approach to the steady state is in a sense monotonic.

5.2. Three angles of one sign and one of the other. For this case, the inequalities (5.1) still hold and two of them are identically satisfied, but the remaining two contain only three of the four angles $\beta_{i}$ and hence cannot be combined to establish (5.2). It follows that we are unable to establish convergence of $\mathcal{C}_{1}$ orbits for this case, and indeed we shall present a counterexample in the next section.

Also, for this case $\mathcal{R}<0$, implying that successive phases of positive slip at node 1 in successive cycles lie on opposite sides of the steady state orbit. We shall refer to this phenomenon as "alternation."

6. Example 1: An unstable $\mathcal{C}_{1}$ orbit. We consider the example

$$
\boldsymbol{A}=\left[\begin{array}{cc}
1.0 & 0.5 \\
0.5 & 1.0
\end{array}\right], \quad \boldsymbol{B}=\left[\begin{array}{cc}
0.5 & 0.25 \\
0.95 & 0.95
\end{array}\right], \quad \text { with } \quad \mu_{1}=\mu_{2}=1
$$

The matrix $\boldsymbol{A}$ is clearly positive definite, and it is easily verified that these values satisfy the conditions (3.4), (3.5). The angles are given by

$$
\beta_{1}=0.464, \beta_{2}=1.460, \beta_{3}=0.464, \beta_{4}=-0.639,
$$

so

$$
\tan \beta_{1}=0.500, \tan \beta_{2}=9.000, \tan \beta_{3}=0.500, \tan \beta_{4}=-0.744,
$$

and

$$
\mathcal{R}=\prod_{1 \leq i \leq 4}\left(\tan \beta_{i}\right)=-1.673
$$

for which $|\mathcal{R}|>1$, indicating instability.

Simple loading conditions were chosen such that each constraint resides at a "rest" position (shown as dashed inclined lines in Figure 6) except that each advances to an extreme position (solid lines in Figure 6) and then recedes, in the sequence $\mathcal{O}_{+}^{1}$ (green), $\mathcal{O}_{+}^{2}$ (blue), $\mathcal{O}_{-}^{1}$ (red), $\mathcal{O}_{-}^{2}$ (maroon). Regions of $v_{1}-v_{2}$-space that are excluded by the 


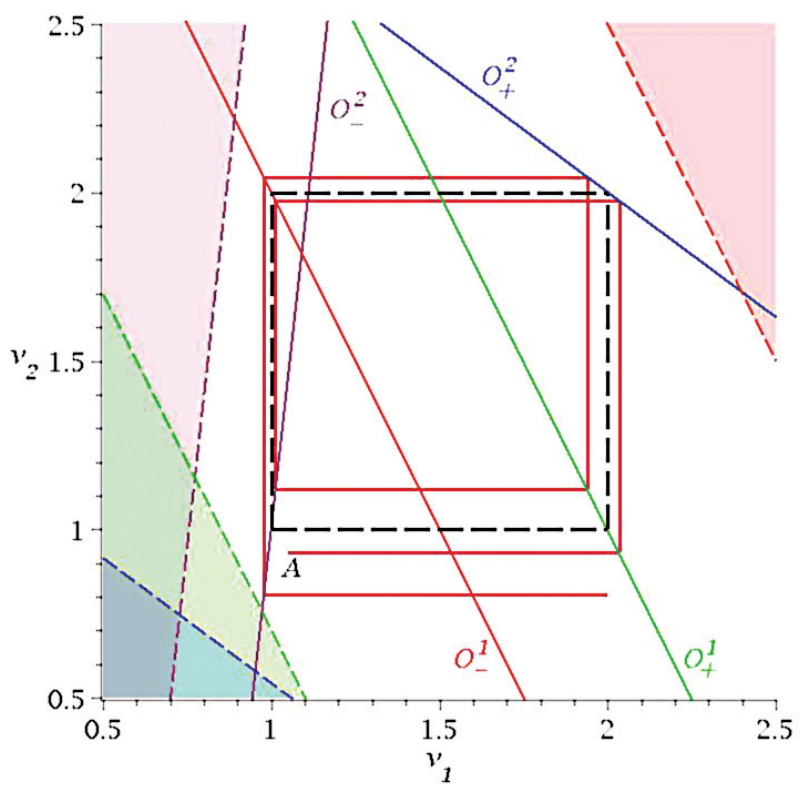

FIG. 6. Transient response for a system with a repelling periodic orbit starting from an initial condition A close to that orbit. Notice how segments governed by a given constraint alternate between inside and outside the repelling orbit.

rest positions of the constraints are shown shaded in this and subsequent figures. Figure 6 shows the resulting rectangular steady-state orbit (black dashed line) and the transient evolution from a position $A$ relatively close to the steady state. This transient was determined using arguments similar to those used in connection with Figure 2, but the results were also confirmed using the numerical algorithm described by Ahn and Barber [1].

Notice that successive cycles deviate further from the steady state. This behavior was obtained from all starting positions, as indeed is implied by condition (5.2). Notice also that during the second cycle, the transient trajectory is on the opposite side of the steady state compared to the trajectory during the first cycle, as implied by the condition $\mathcal{R}<0$. This alternation continues as long as condition (i) of Definition 3.1 remains satisfied.

6.1. Limit cycle. When a periodic orbit in class $\mathcal{C}_{1}$ is unstable, deviations from it grow exponentially until a condition is reached at which condition (i) of Definition 3.1 is violated. In other words, a situation is reached where two constraints are simultaneously active. In the example of Figure 6, this occurs when the motion $\dot{v}_{2}<0$ intersects the rest position of constraint $\mathcal{O}_{+}^{1}$. At this point, the trajectory immediately achieves the stable limit cycle illustrated in Figure 7, which involves a period of simultaneous slip $\dot{v}_{1}>0, \dot{v}_{2}<0$ at both nodes. The numerical code was used to track the evolution of this system from a range of initial conditions, all of which converged on this limit cycle after a finite number of loading cycles. Notice that because of the alternation discussed above, the limit cycle extends over two cycles of the external loading.

Extensive exploration of the transient behavior of this example suggest that this same limit cycle is achieved for all initial conditions. 


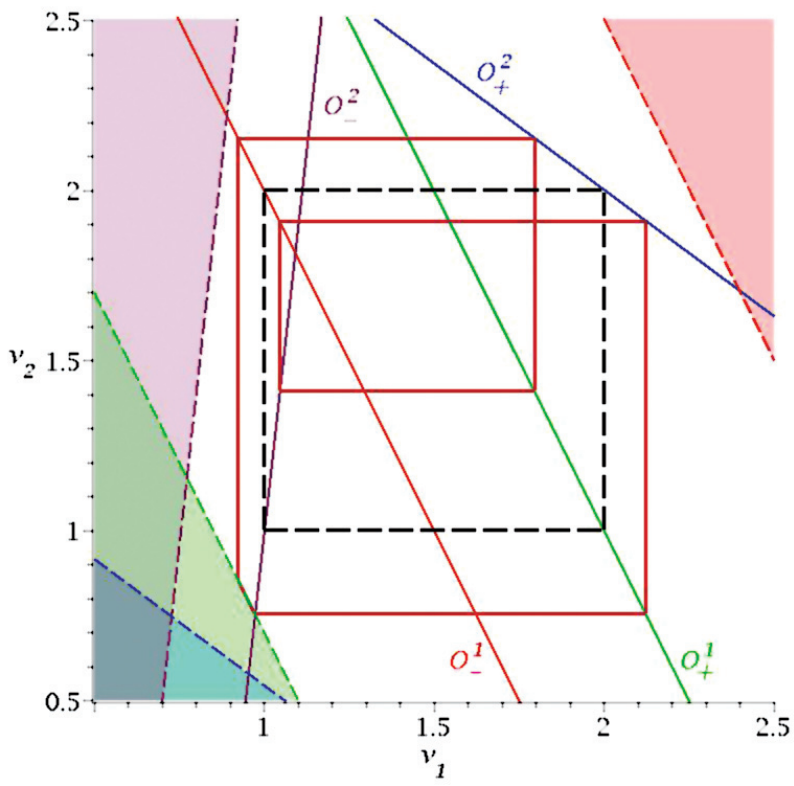

FIG. 7. The limit cycle for Example 1 (orange). The dashed black line defines the unstable rectangular orbit.

7. More complex orbits. We next define a broader class of loading conditions $\mathcal{C}_{2}$ such that condition (ii) of Definition 3.1 is retained, but (i) is relaxed-i.e., we permit cases where there exists a steady state in which one or more of the constraints is active multiple times in each cycle.

The most general orbit in $\mathcal{C}_{2}$ involves the action of the four constraints in any sequence, with any number of segments. For example, we might have

$$
\mathcal{O}_{+}^{1} \mathcal{O}_{-}^{2} \mathcal{O}_{-}^{1} \mathcal{O}_{+}^{2} \mathcal{O}_{-}^{2} \mathcal{O}_{+}^{1} \mathcal{O}_{+}^{2} \mathcal{O}_{-}^{1} \mathcal{O}_{+}^{1} \mathcal{O}_{-}^{1} \mathcal{O}_{-}^{2} \mathcal{O}_{+}^{1} \cdots
$$

repeating from this point onwards. However, if any segment is followed by slip in the opposite direction at the same node, we see from Figure 5 and (4.1) that there is no change in $\Delta$ and the final state is the same as if only the second of the two slip segments had been present. Thus, for the purpose of calculating the change in $\Delta$ during a complete cycle, the above sequence can be condensed to

$$
\mathcal{O}_{+}^{1} \mathcal{O}_{-}^{2} \mathcal{O}_{-}^{1} \mathcal{O}_{-}^{2} \mathcal{O}_{+}^{1} \mathcal{O}_{+}^{2} \mathcal{O}_{-}^{1} \mathcal{O}_{-}^{2} \mathcal{O}_{+}^{1} \cdots
$$

It then follows that during one loading cycle, the initial value of $\Delta$ is multiplied by the ratio

$$
\begin{aligned}
\mathcal{R} & =\left(-\tan \beta_{1}\right)\left(\tan \beta_{2}\right)\left(-\tan \beta_{3}\right)\left(\tan \beta_{2}\right)\left(-\tan \beta_{1}\right)\left(\tan \beta_{4}\right)\left(-\tan \beta_{3}\right)\left(\tan \beta_{2}\right) \\
& =\left(-\tan \beta_{1}\right)^{2}\left(\tan \beta_{2}\right)^{3}\left(-\tan \beta_{3}\right)^{2}\left(\tan \beta_{4}\right) .
\end{aligned}
$$

Notice that each of the factors in this expression defines a transition either from slip at node 1 to node $2\left(\beta_{1}, \beta_{3}\right)$ or from node 2 to node $1\left(\beta_{2}, \beta_{4}\right)$. The number of these transitions must clearly be equal, so for the most general orbit in $\mathcal{C}_{2}$, we shall have

$$
\mathcal{R}=\left(-\tan \beta_{1}\right)^{p}\left(\tan \beta_{2}\right)^{q}\left(-\tan \beta_{3}\right)^{r}\left(\tan \beta_{4}\right)^{s}
$$


under the restriction

$$
p+r=q+s .
$$

The stability criterion $|\mathcal{R}|<1$ will be satisfied for all $p, q, r, s$ and hence for all loading cycles in class $\mathcal{C}_{2}$ if and only if

$$
\max \left(\left|\tan \beta_{1}\right|,\left|\tan \beta_{3}\right|\right)<\min \left(\left|\cot \beta_{2}\right|, \cot \left|\beta_{4}\right|\right) .
$$

If this is not satisfied, so that there exist two values (say $\beta_{1}, \beta_{2}$ ) such that

$$
\left|\tan \beta_{1}\right|>\left|\cot \beta_{2}\right| \quad \text { or } \quad\left|\tan \beta_{1} \tan \beta_{2}\right|>1,
$$

then we can choose $r=s=1$ and $p=q$ to obtain

$$
|\mathcal{R}|=\left|\left(\tan \beta_{1} \tan \beta_{2}\right)^{q}\left(\tan \beta_{3}\right)\left(\tan \beta_{4}\right)\right|,
$$

and this can be made to exceed unity by choosing $q$ sufficiently large. Inequality (7.2) can also be written in the alternative form

$$
\left|\tan \beta_{2}\right|>\left|\tan \alpha_{2}\right|
$$

using (3.1), and hence, in view of (3.6), (3.7),

$$
\left|\beta_{2}\right|>\alpha_{2} \quad \text { or } \quad\left|\beta_{2}\right|>\pi-\alpha_{2} .
$$

Thus, unstable orbits can exist if and only if at least one of the four points $\alpha_{i}, \beta_{i}$ lies in one of the triangles $A B F$ and $C D E$ in Figure 4.

We also note that if

$$
\mathcal{R}<0,
$$

the transient trajectory will alternate. If the orbit is also unstable, we anticipate that there will be a unique limit cycle that involves two loading cycles, as in the example in section 6 .

If $\mathcal{R}>0$, the trajectory will approach or diverge from the periodic orbit monotonically, and in cases of instability $(\mathcal{R}>1)$ we anticipate two distinct limit cycles, the one reached depending on which side of the unstable orbit the initial condition is on. We present an example of this kind in the next section.

8. Example 2: An unstable $\mathcal{C}_{2}$ orbit with two limit cycles. We consider an example using the same stiffness matrices as in section 6 and coefficients of friction $\mu_{1}=\mu_{2}=0.98$. However, the loading cycle was modified such that constraints $\mathcal{O}_{+}^{1}$ and $\mathcal{O}_{-}^{2}$ were active twice per cycle, the other constraints being active only once. The constraints are active in the order $\mathcal{O}_{-}^{2}, \mathcal{O}_{+}^{1}, \mathcal{O}_{-}^{2}, \mathcal{O}_{+}^{1}, \mathcal{O}_{+}^{2}, \mathcal{O}_{-}^{1}$, and the corresponding steady-state orbit is shown as a dashed black line in Figure 8. It is traversed in the counterclockwise direction and is unstable with

$$
\mathcal{R}=3.614 \text {. }
$$

Since $\mathcal{R}>0$, the transient response remains on the same side of the steady state in successive cycles, and a different limit cycle is obtained depending on the initial perturbation. The orange limit cycle in Figure 8 corresponds to the case where the initial point is slightly to the left of the top left corner of the steady state orbit and, 


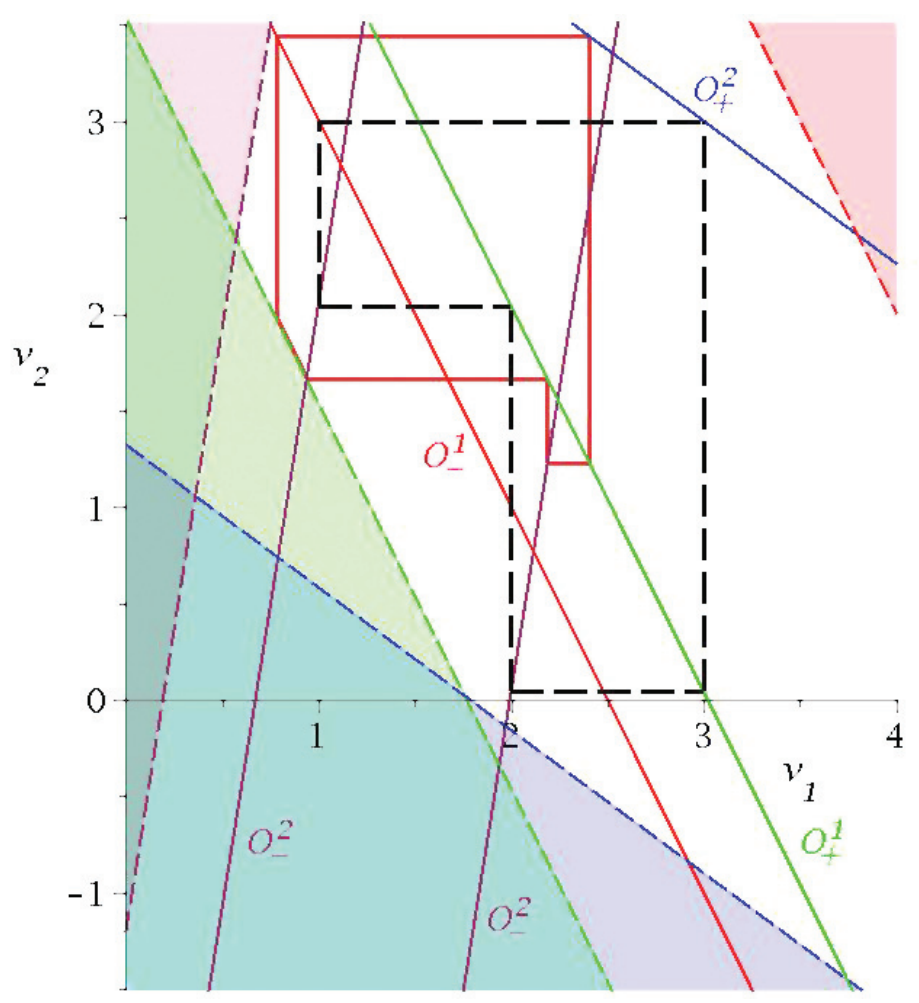

FIG. 8. First limit cycle for Example 2.

as in Example 1, the limiting process comprises the occurrence of slip at both nodes simultaneously, due to the intersection of a segment of slip governed by $\mathcal{O}_{-}^{2}$ (maroon) with the rest position of $\mathcal{O}_{+}^{1}$ (green).

If instead we choose an initial condition slightly to the right of the top left corner, the first violation of condition (i) occurs when a segment of slip governed by $\mathcal{O}_{-}^{2}$ intersects with the rest position of $\mathcal{O}_{+}^{2}$ (i.e., the other constraint for the same node). For these two constraints to be active simultaneously, it is necessary that $p_{2}$ should be zero, showing that this intersection defines the beginning of a segment of separation at node 2 . In this case, when $\mathcal{O}_{-}^{2}$ recedes, the node will re-establish contact at a point that (i) lies on the same horizontal line (since no slip occurs at node 1 throughout this process) and (ii) lies on both $\mathcal{O}_{+}^{2}$ and $\mathcal{O}_{-}^{2}$. Since we have assumed loading conditions such that $\mathcal{O}_{+}^{2}$ remains stationary during this phase of the loading, this point is uniquely defined by condition (i) and $\mathcal{O}_{+}^{2}$, and hence contact is re-established at the same point at which it separated.

In contrast to the procedure defining the limit cycle of Figures 6 and 7, this intersection is not unique, since it depends on the value of $v_{1}$ during the preceding slip segment and hence also on the initial condition. However, once a period of separation has occurred, a further evolution of the trajectory occurs, governed by the rest position of $\mathcal{O}_{+}^{2}$ instead of the extreme position of $\mathcal{O}_{-}^{2}$. This has the same effect as a reduction of the ratio $\mathcal{R}$ to a value less than unity, and hence the trajectory tends exponentially to a limit cycle involving a period of separation at node 2 . This limit cycle is shown in orange in Figure 9. Notice that the short vertical extension of the 


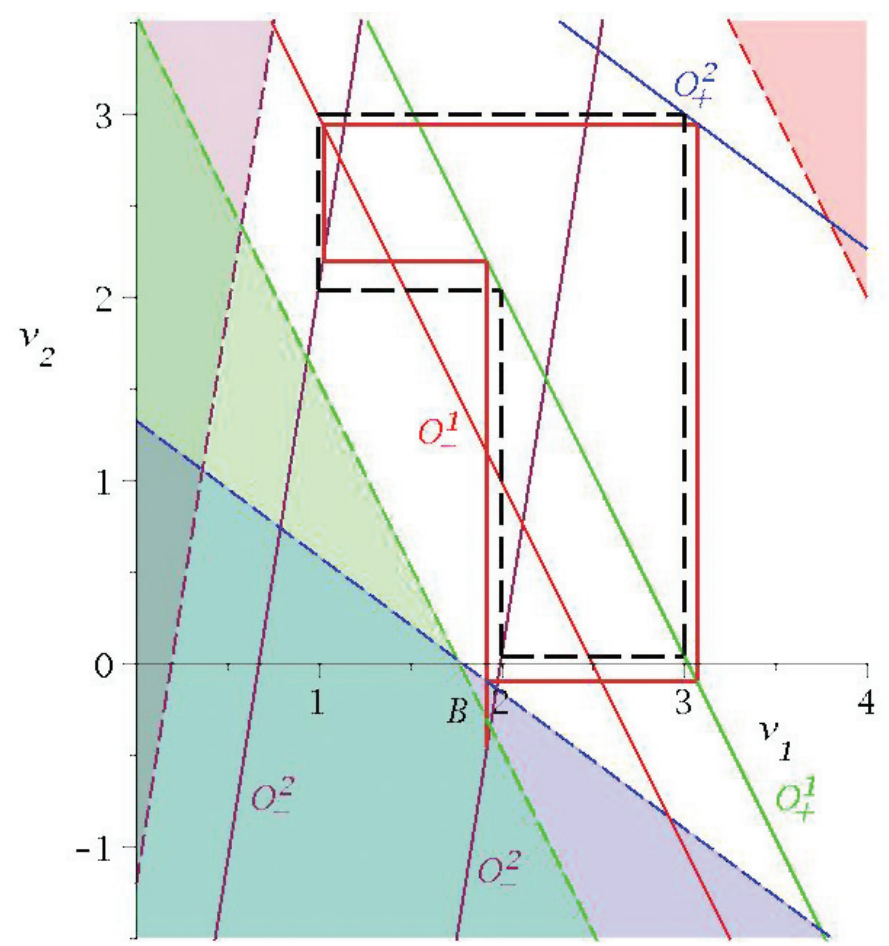

FiG. 9. Second limit cycle for Example 2.

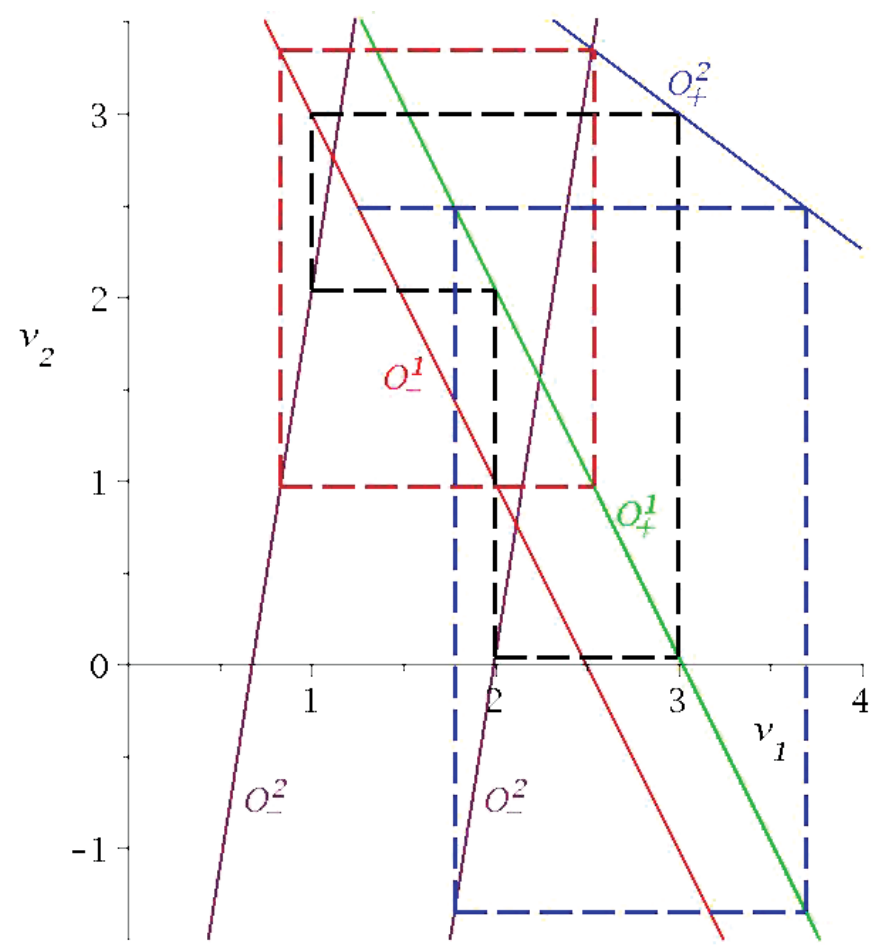

FIG. 10. Stable orbits in class $\mathcal{C}_{2}$ for Example 2. 
orbit near the corner $B$ represents the tangential displacement that occurs (out and back) during the period of separation at node 2 .

8.1. Limiting behavior remaining in $\mathcal{C}_{2}$. Examples of class $\mathcal{C}_{2}$ can reach limiting orbits without violating condition (ii) of Definition 3.1 if the rest positions of the constraints are sufficiently far removed as to remain inactive. We first note that if the second advance of $\mathcal{O}_{+}^{1}$ is temporarily disregarded, we can construct a stable rectangular orbit, which is shown as the dashed orange line in Figure 10. Furthermore, if the system reaches this orbit, the second advance of $\mathcal{O}_{+}^{1}$ will indeed not cause any slip, so this orbit is a stable limit cycle for the system. In this case (with sufficiently distant rest positions), initial conditions slightly to the left of the top corner of the unstable black orbit are repelled from this orbit until the divergence is sufficient to render the second advance of $\mathcal{O}_{+}^{1}$ inactive, after which the system is attracted to the rectangular orange orbit.

A starting point slightly to the right of the top left corner of the black orbit leads to a different situation where eventually the first advance of $\mathcal{O}_{+}^{1}$ becomes inactive and the segment of negative slip at node 1 is followed by a segment of positive slip at the same node. This orbit is represented by the dashed blue line in Figure 10, and it is also stable.

9. An example of nonuniqueness for the quasi-static evolution problem. Using a construction like that in section 7 we will show that the condition (4.6) is also closely connected to the question of uniqueness for the quasi-static evolution problem.

We will give an example of an elastic system for which the rate problem has a unique solution for all loadings, but where there exists a time-dependent external loading $-\boldsymbol{f}(t)=\left(\boldsymbol{q}^{w}(t), \boldsymbol{p}^{w}(t)\right)^{T}$ with $\boldsymbol{f} \in C^{\infty}[0, \infty)$ such that the evolution problem has infinitely many solutions.

A similar nonuniqueness result has been given previously by Ballard [4]. The nonuniqueness example in Ballard's paper is for a one-node system with three spatial degrees of freedom and with an initial state with so-called grazing contact, i.e., with the initial reaction force being equal to zero. For the example that we shall present, the number of nodes is two, and the spatial degrees of freedom are two. Further, we consider a loading where we have full contact, i.e., with a strictly positive normal contact force at all times. A significant difference between the examples is that Ballard shows that a counterexample exists for arbitrarily small coefficients of friction. Our analysis shows that there exists an elastic systems with two nodes and two spatial dimensions such that all rate problems are uniquely solvable, but that there still exist multiple solutions to the time evolution problem.

There are also some similarities regarding the construction of the applied force. In Ballard's paper an applied force is used where the tangential component $\boldsymbol{f}_{T}(t)$ changes direction at $t \rightarrow 0^{+}$, i.e., so that the normalized vector $\boldsymbol{f}_{T}(t) /\left|\boldsymbol{f}_{T}(t)\right|$ has no limit (although $\boldsymbol{f}_{T}(t)$ has a bounded time derivative). In our paper the external force field is constructed so that one changes between stick at node 1, slip at node 2, and, conversely, stick at node 2 , slip at node 1 infinitely many times as $t \rightarrow 0^{+}$. The force field and the resulting displacement field are $C^{\infty}$ in time.

The present counterexample shows that imposing conditions of full contact (nongrazing contact) and restriction to two spatial dimensions and systems where all rate problems are uniquely solvable is not enough to guarantee uniqueness for the time evolution problem.

Assume that we have an elastic system with two nodes and two spatial dimensions such that the conditions (3.4) and (3.5) guaranteeing that all rate problems are unique- 
ly solvable are valid, but that the condition 4.6 is violated so that, for example, $\cos \alpha_{1} / \cos \beta_{1}>1$.

Let times $\left\{t_{i}\right\}_{i=-\infty}^{\infty}$, with $t_{i}<t_{i+1}$, be given. Then we can alternatingly apply the obstacles $\mathcal{O}_{+}^{1}$ and $\mathcal{O}_{+}^{2}$ to two different initial states in the following way. First, for all $t_{2 n+1}$ the obstacles $\mathcal{O}_{+}^{2}\left(t_{2 n+1}\right)$ will have the same position. Next, considering an interval $t_{2 n}<t<t_{2 n+2}$ the obstacles have for $t=t_{2 n}$ the positions $\mathcal{O}_{+}^{1}\left(t_{2 n}\right)$ and $\mathcal{O}_{+}^{2}\left(t_{2 n}\right)$ as shown in Figure 11, and we consider the initial states which are the intersections between $\mathcal{O}_{+}^{2}\left(t_{2 n}\right), \mathcal{O}_{+}^{1}\left(t_{2 n}\right)$ (blue point) and $\mathcal{O}_{+}^{2}\left(t_{2 n+1}\right), \mathcal{O}_{+}^{1}\left(t_{2 n}\right)$ (red point), respectively.

Then, while $\mathcal{O}_{+}^{1}$ remains constant, $\mathcal{O}_{+}^{2}$ advances to the position $\mathcal{O}_{+}^{2}\left(t_{2 n+1}\right)$ and then recedes to the position $\mathcal{O}_{+}^{2}\left(t_{2 n+2}\right)$, which is reached for some $t^{\prime}<t_{2 n+2}$, and remains constant for $t^{\prime}<t<t_{2 n+2}$. It follows that the blue point is moved vertically to a new position. Then the obstacle $\mathcal{O}_{+}^{1}$ moves to a position $\mathcal{O}_{+}^{1}\left(t_{2 n+2}\right)$, which is reached for some $t^{\prime \prime}<t_{2 n+2}$, and $\mathcal{O}_{+}^{1}(t)$ is constant for $t^{\prime \prime}<t<t_{2 n+2}$. It follows that the blue point will move horizontally to a new position. Further, the position is chosen such that both the red point and the blue point (new positions) are positions on the obstacle $\mathcal{O}_{+}^{1}\left(t_{2 n+2}\right)$. Consequently the red and blue initial points have moved to new positions such that their distance have been multiplied by a factor

$$
\frac{\cos \alpha_{1}}{\cos \beta_{1}} \frac{\sin \beta_{1}}{\sin \alpha_{1}}=\tan \beta_{1} \tan \beta_{4}:=d^{2}>1
$$

This procedure is repeated for all intervals $\left(t_{2 n}, t_{2 n+2}\right)$ giving the positions of the obstacles as in Figure 11.

Writing the equations of the obstacles as

$$
\begin{aligned}
& \left(a_{11}+\mu_{1} b_{11}\right) v_{1}+\left(a_{12}+\mu_{1} b_{12}\right) v_{2}=s_{1}(t)=-\mu_{1} p_{1}^{w}(t)-q_{1}^{w}(t), \\
& \left(a_{21}+\mu_{1} b_{21}\right) v_{1}+\left(a_{22}+\mu_{1} b_{22}\right) v_{2}=s_{2}(t)=-\mu_{1} p_{2}^{w}(t)-q_{2}^{w}(t),
\end{aligned}
$$

we have $s_{2}\left(t_{2 n+1}\right)=s_{2}\left(t_{-\infty}\right)$ independent of $n$. Further, denoting

$$
\lim _{n \rightarrow-\infty} s_{i}\left(t_{n}\right)=s_{i}\left(t_{-\infty}\right),
$$

we have for $i=1,2$ and all $n$,

$$
\left(s_{i}\left(t_{2 n+4}\right)-s_{i}\left(t_{2 n+2}\right)\right) /\left(s_{i}\left(t_{2 n+2}\right)-s_{i}\left(t_{2 n}\right)\right)=d^{2}>1
$$

and

$$
\left(s_{i}\left(t_{2 n+2}\right)-s_{i}\left(t_{-\infty}\right)\right) /\left(s_{i}\left(t_{2 n}\right)-s_{i}\left(t_{-\infty}\right)\right)=d^{2} .
$$

In order to proceed we need to specify in detail the time parametrization of the external force field over all the intervals $\left(t_{2 n}, t_{2 n+2}\right)$.

Let $\mathcal{A}_{1}$ and $\mathcal{A}_{2}$ be functions in $C^{\infty}[0,1]$ with the general properties depicted in Figure 12, i.e., $-\mathcal{A}_{2}(t)=1$ if $t \in\left[0, \tau_{1}\right]$ and $-\mathcal{A}_{2}(t)=d^{2}$ if $t \in\left[\tau_{4}, 1\right], \mathcal{A}_{2}(t)=0$ if $t \in\left[\tau_{2}, \tau_{3}\right]$, and $\mathcal{A}_{2}$ is decreasing and increasing, respectively, on $\left[\tau_{1}, \tau_{2}\right]$ and $\left[\tau_{3}, \tau_{4}\right]$. Similarly $\mathcal{A}_{1}(t)=1$ if $t \in\left[0, \tau_{4}\right], \mathcal{A}_{1}(t)=d^{2}$ if $t \in\left[\tau_{5}, 1\right]$, and $\mathcal{A}_{1}$ is increasing on $\left[\tau_{4}, \tau_{5}\right]$. Further, $\tau_{4}=\tau^{\prime}$ and $\tau_{5}=\tau^{\prime \prime}$, respectively, correspond to the values $t^{\prime}$ and $t^{\prime \prime}$ above. we take

Now, for $n \geq 2$ we take $t_{-2 n}=1 / n$. Then we have $t_{-\infty}=0$ and, for $t \in\left[\frac{1}{n}, \frac{1}{n-1}\right]$,

$$
s_{2}(t)-s_{2}(0)=C d^{-2 n} \mathcal{A}_{2}(n(n-1)(t-1 / n)),
$$




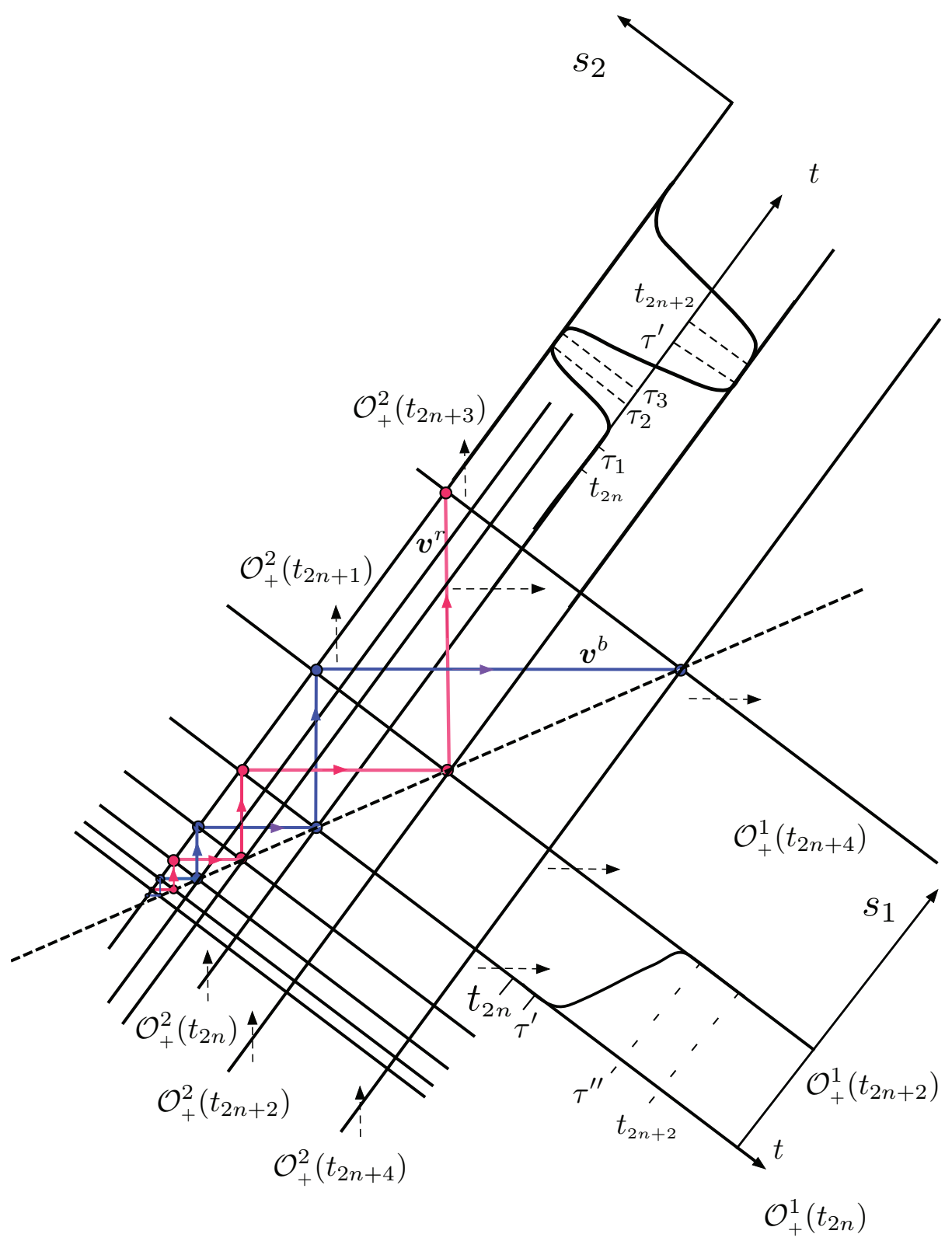

FIG. 11. Nonunique solutions.

where $C$ is some constant. Here $\frac{1}{n(n-1)}$ is the length of the interval $\left[\frac{1}{n}, \frac{1}{n-1}\right]$ and $\mathcal{A}_{2}(n(n-1)(t-1 / n))$ a translated and dilated version of the function $\mathcal{A}_{2}$. Taking $n=2, t=1$, we get

$$
s_{2}(1)-s_{2}(0)=C d^{-4} \mathcal{A}_{2}(1)=-C d^{-2},
$$

whence we conclude that we must choose $C=d^{2}\left(s_{2}(1)-s_{2}(0)\right)$. Therefore we choose

$$
s_{2}(t)-s_{2}(0)=\left(s_{2}(1)-s_{2}(0)\right) d^{2(1-n)} \mathcal{A}_{2}(n(n-1)(t-1 / n))
$$



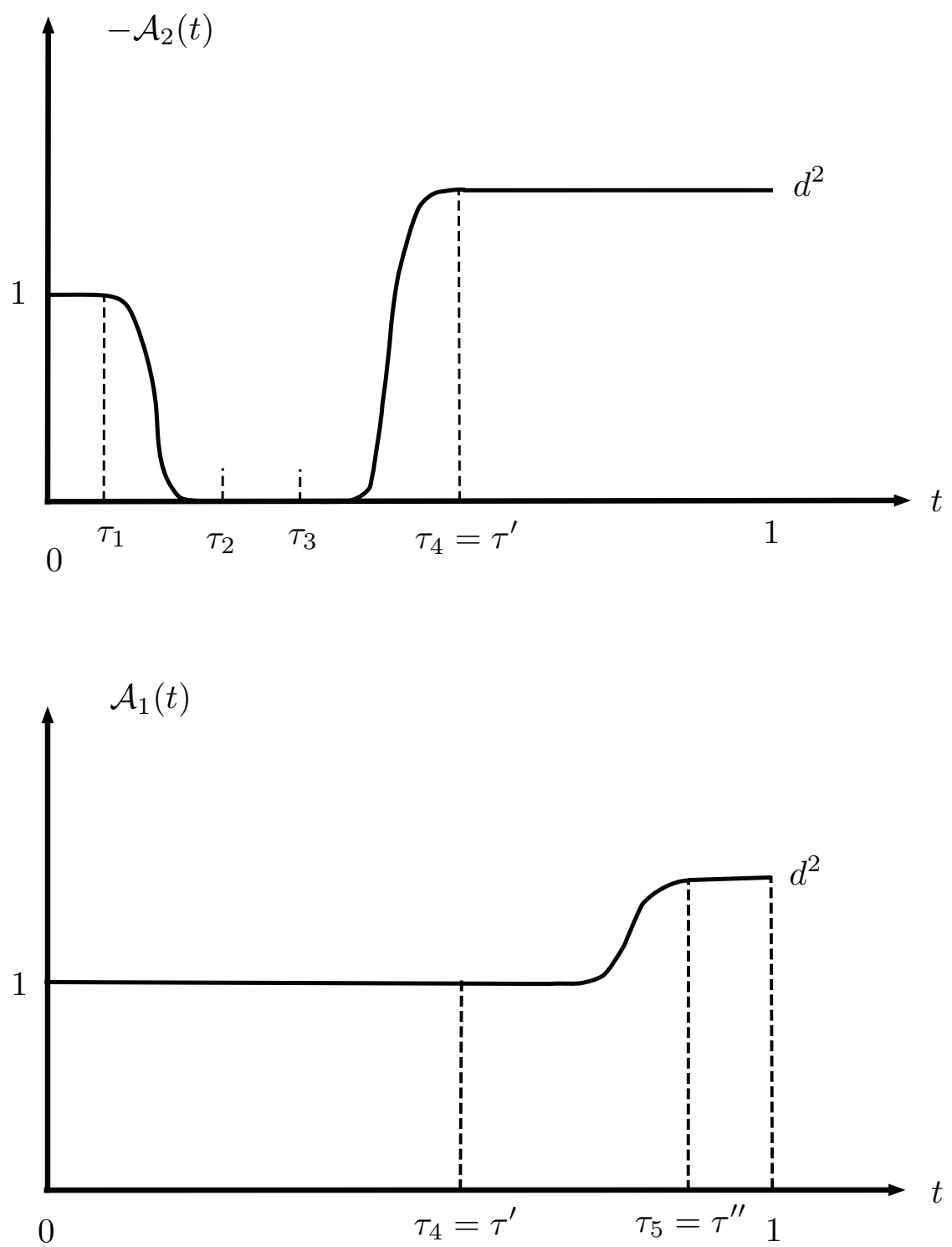

FIG. 12. Auxiliary functions used to define external force functions of the counterexample.

if $t \in\left[\frac{1}{n}, \frac{1}{n-1}\right]$. Similarly we define

$$
s_{1}(t)-s_{1}(0)=\left(s_{1}(1)-s_{1}(0)\right) d^{2(1-n)} \mathcal{A}_{1}(n(n-1)(t-1 / n)) .
$$

It is clear that $s_{i} \in C^{\infty}(0,1]$. For the $k$ th derivative we get

$$
s_{i}^{(k)}(t)=\left(s_{i}(1)-s_{i}(0)\right) d^{2(1-n)} \mathcal{A}_{i}^{(k)}(n(n-1)(t-1 / n)) n^{k}(n-1)^{k}
$$

if $t \in\left[\frac{1}{n}, \frac{1}{n-1}\right]$. Since the $k$ th derivatives of $\mathcal{A}_{i}$ are bounded on [0,1], we conclude that for some constant $C$,

$$
\left|s_{i}^{(k)}(t)\right| \leq C d^{-2 n} n^{2 k} \quad \text { if } \quad t \in\left[\frac{1}{n}, \frac{1}{n-1}\right] .
$$


Since $\lim _{n \rightarrow \infty} n^{2 k} / d^{2 n} \rightarrow 0$ as $n \rightarrow \infty$, it follows that $\lim _{t \rightarrow 0} s_{i}^{(k)}(t)=0$ for every $k$ th derivative, i.e., that $s_{i} \in C^{\infty}[0,1]$.

Finally, in order to define the external force field, we use (9.1) and (9.2), giving

$$
\begin{aligned}
& q_{1}^{w}(t)=-\mu_{1} p_{1}^{w}(t)-s_{1}(t), \\
& q_{2}^{w}(t)=-\mu_{1} p_{2}^{w}(t)-s_{2}(t) .
\end{aligned}
$$

Now we may, for example, choose $p_{1}^{w}(t)$ and $p_{2}^{w}(t)$ to be constant and large enough (to ensure that contact is not lost) and choose $q_{1}^{w}(t)$ and $q_{2}^{w}(t)$ according to (9.3) and (9.4). Then we have defined an external force field $\boldsymbol{f}(t)$ which is in $C^{\infty}[0,1]$. Further, the evolution problem with initial conditions $\boldsymbol{v}(0), \boldsymbol{f}(0)$ given by

$$
\begin{aligned}
& \left(a_{11}+\mu_{1} b_{11}\right) v_{1}(0)+\left(a_{12}+\mu_{1} b_{12}\right) v_{2}(0)=s_{1}(0), \\
& \left(a_{21}+\mu_{1} b_{21}\right) v_{1}(0)+\left(a_{22}+\mu_{1} b_{22}\right) v_{2}(0)=s_{2}(0)
\end{aligned}
$$

and

$$
\begin{aligned}
& q_{1}^{w}(0)=-\mu_{1} p_{1}^{w}-s_{1}(0), \\
& q_{2}^{w}(0)=-\mu_{1} p_{2}^{w}-s_{2}(0)
\end{aligned}
$$

has two different solutions $\boldsymbol{v}^{b}(t)$ and $\boldsymbol{v}^{r}(t)$, infinitely differentiable for $0 \leq t \leq 1$ and satisfying the same initial condition for $t=0$ and the rate problem for all $t$. Their orbits are shown in Figure 11 colored in blue and red. It is also easy to see that there are infinitely many solutions intermediate to the red and blue ones.

We end this section with a remark.

Remark 9.1. It was shown in [3] that one has uniqueness for the evolution problem, provided that

- the coefficients of friction were small enough for the rate problem to be uniquely solvable, in our special case such that the conditions (3.4) and (3.5) are satisfied,

- the force field is a piecewise real analytic function of time

This condition of an analyticity rules out the occurrence of infinitely many oscillations that are present in the previous example on nonuniqueness.

10. Conclusions. In previous papers, it was conjectured (based on heuristic arguments involving the concept of system memory) that in discrete frictional elastic systems where all nodes slip at least once during each cycle of periodic loading, there would exist a unique steady state to which orbits from all initial conditions would be attracted. In this paper, we have disproved this conjecture by demonstrating the existence of counterexamples in the context of a simple two-node system. In particular, there can exist steady-state orbits that are unstable in the sense that they repel orbits starting from initial conditions that are sufficiently close to the steady state.

Criteria for determining the stability of a steady state have been developed, and these depend only on the loading sequence and on the slopes of the frictional constraint lines, defined in terms of the stiffness matrix and the coefficient(s) of friction. In particular we have shown these criteria are, in general, more restrictive than those which guarantee that the rate problem is uniquely solvable for arbitrary derivative of the external loading. Further, the problem of establishing nonexistence of unstable steady states is closely connected to the problem of establishing uniqueness for the quasi-static evolution problem. An example of an elastic system is given, where there 
exists a loading with an unstable periodic orbit, as well as a loading with multiple (infinitely many) solutions of a quasi-static problem.

A commonly occurring state leading to periodic frictional slip is that in which the contact nodes are subjected to a set of mean loads and a superposed sinusoidal oscillation due to machine vibration at a single frequency. Under these conditions, only one segment of slip can occur per cycle at each node in a given direction, and unstable orbits for the two-node system can occur only when the slopes of the constraints satisfy the condition established in section 5.2. The resulting transient orbit will then alternate on the two sides of the steady state as it evolves.

In cases of instability, the transient orbit is eventually limited either by slip occurring at both nodes simultaneously, or by one node separating. In both cases the system eventually reaches a stable limit cycle. Depending on the slopes of the constraint lines, the limit cycle can involve two periods of the loading cycle, in which case it appears to be unique, or it may repeat every loading cycle, in which case distinct limit cycles are reached depending on the sign of the initial deviation from the steady state.

\section{REFERENCES}

[1] Y. J. Ahn And J. R. Barber, Response of frictional receding contact problems to cyclic loading, Internat. J. Mech. Sci., 50 (2008), pp. 1519-1525.

[2] Y. J. Ahn, E. Bertocchi, And J. R. BARBer, Shakedown of coupled two-dimensional discrete frictional systems, J. Mech. Phys. Solids, 56 (2008), pp. 3433-3440.

[3] L.-E. Andersson, Quasistatic Frictional Contact Problems with Finitely Many Degrees of Freedom, Technical report LiTH-MAT-R-1999-22, Linköping University, 1999.

[4] P. Ballard, A counterexample to uniqueness in quasi-static elastic contact problems with small friction, Internat. J. Engrg. Sci., 37 (1999) pp. 163-178.

[5] J. R. BARBer, Frictional systems subjected to oscillating loads, Ann. Solid Struct. Mech., 2 (2011), pp. 45-55.

[6] Y. H. JANG AND J. R. BARBER, Frictional energy dissipation in materials containing cracks, J. Mech. Phys. Solids, 59 (2011), pp. 583-594.

[7] K. L. Johnson, Contact Mechanics, Cambridge University Press, Cambridge, UK, 1985, sect. 7.2 .

[8] A. KlaRBRIng, Contact, friction, discrete mechanical structures and discrete frictional systems and mathematical programming, in New Developments in Contact Problems, P. Wriggers and P. Panagiotopoulos, eds., Springer, New York, 1999, pp. 55-100.

[9] A. Klarbring, M. Ciavarella, and J. R. Barber, Shakedown in elastic contact problems with Coulomb friction, Internat. J. Solids Structures, 44 (2007), pp. 8355-8365.

[10] D. Nowell, D. Dini, And D. A. Hills, Recent developments in the understanding of fretting fatigue, Eng. Fract. Mech., 73 (2006), pp. 207-222.

[11] H. Wentzel, Modelling of Frictional Joints in Dynamically Loaded Structures: A Review, Technical report, KTH Solid Mechanics, Royal Institute of Technology, 2006. 